\title{
Implementation of envelope detection based Wake-Up Receiver for IEEE 802.15.4 WPAN from off-the-shelf components
}

\author{
Josua Arndt, Lukas Krystofiak, Vahid Bonehi, Ralf Wunderlich, and Stefan Heinen \\ RWTH Aachen University, Integrated Analog Circuits and RF Systems, 52074 Aachen, Germany \\ Correspondence to: Josua Arndt (jarndt@ias.rwth-aachen.de)
}

Received: 2 November 2016 - Revised: 1 March 2017 - Accepted: 22 April 2017 - Published: 21 September 2017

\begin{abstract}
Power consumption in wireless networks is crucial. In most scenarios the transmission time is short compared to the idle listening time for data transmission, the most power is consumed by the receiver. In low latency systems there is a need for low power wake-up receivers (WuRx) that reduce the power consumption when the node is idle, but keep it responsive. This work presents a WuRx designed out of commercial components to investigate the needs of a WuRx when it is embedded in a Wireless Personal Area Network (WPAN) system in a real environment setup including WLAN and LTE communication and considering interferer rejection. The calculation necessary for the attenuation of those interferers is explained in detail. Furthermore, a system design is presented that fulfills the requirements for this environment and is build from off-the-shelf components.
\end{abstract}

\section{Introduction}

Personal Area Networks, as specified by (Gutierrez et al., 2003), applications like lighting control, monitoring temperature, moisture etc. or IoT (Internet of Things) devices can demand a fast reaction in lighting systems for example. If the user activates the light it should react in milliseconds as we are used to the lights switching on instantly. For those applications, the receiver must always be active to listen to the channel. In beacon-enabled networks a fast reaction time can be achieved by a big duty-cycle, resulting in a high beacon number to send. The end devices must wake up frequently which results in a high-energy consumption. A WuRx designed to support a low data rate modulation can be very simple and consumes less energy than a high data rate modulation like BPSK or O-QPSK.
Conventional receiver architectures are the heterodyne or homodyne (zero-IF) receiver which support complex modulation schemes whereby a high spectral efficiency and at last a high data rate can be achieved. Both rely on at least one LO (Local Oscillator) which commonly consists of a LC oscillator embedded in a power consuming PLL (phase-locked loop) with an operating frequency in the RF range. A regenerative circuit as alternative receiver concept is tempting because it only oscillates when there is a signal at the input, but the bias current of the of the LC circuit is the main problem in reducing power dissipation. Last there is the direct conversion by an envelope detector, which shows potential for a very low power consumption.

A WuRx using the conventional heterodyne receiver concept architecture is presented in (Pletcher et al., 2008), the power consumption splits up as follows: RF Amplification 22, LO 20, Mixer 8 and the ED $1 \mu \mathrm{W}$. A LO-less approach using a double-sampling technique to suppress the offset and $1 / f$ noise of the down-converting envelope detector is presented in (Huang et al., 2010). The power consumption can be divided as follows: LNA $27 \mu \mathrm{W}$, BB-amplifier $14 \mu \mathrm{W}$, ED $5 \mu \mathrm{W}$, bias circuit $4 \mu \mathrm{W}$, frequency divider and logic $3 \mu \mathrm{W}$, external reference clock $1 \mu \mathrm{W}$. In (Hambeck et al., 2011) a direct down conversion concept is shown, the power consumption on this receiver is as follows: ED $1.25 \mu \mathrm{W}, \mathrm{BB}-$ amplifier $0.4 \mu \mathrm{W}$, correlation unit $0.4 \mu \mathrm{W}$ and the RC lowpass filter $0.2 \mu \mathrm{W}$. Comparing the power dissipation of these wake-up receivers shows that a very low power dissipation can be achieved with the direct conversion approach.

In this paper, we present a work that was done to gain insight for an integrated design, that is developed in parallel. The approach to build a PCB based WuRx which is as near as possible to the targeted system design is followed to enable measurements of the overall system behavior. The PCB 
based WuRx was also developed to be used as a first prototype to implement a full functioning IEEE 802.15.4 network. The Development of on SOC with design, implementation, tape-out and design of a measurement PCB, debug and measure the SOC and develop an application PCB takes up to 3 years. Therefore, a WuRx out of commercial components is built on PCB to investigate the needs of a system that includes a WuRx and uses the IEEE 802.15.4 transmitter to generate the wake-up frame. Challenges will be discussed and solutions presented.

As IEEE 802.15.4 transmitter we used the AT86RF233 from (Atmel, 2014) which is a high-performance RF-CMOS 2.4 GHz radio transceiver targeted for IEEE 802.15.4, ZigBee, RF4CE, 6LoWPAN, and ISM applications. It has a receiver sensitivity of $-101 \mathrm{dBm}$ and a programmable TX output power from -17 to $4 \mathrm{dBm}$.

In Sect. 2 an overview of the requirements will be given and basics of path loss and link margin calculations are presented. In Sect. 3 the system setup, filter design and resulting link budget are presented. In Sect. 4 the measurements of the WuRx are shown and Sect. 5 concludes the paper.

\section{Design requirements}

As the comparison in (Oetting, 1979) shows, on-off-keying (OOK) is the simplest modulation scheme for digital radio transmission. A tuned RF receiver is one of the simplest architectures (Griggs, 2012). It accepts incoming RF signals which are filtered, amplified and converted from RF to baseband by an envelope detector (ED). This eliminates the need for a power consuming local oscillator (LO) completely, which is usually the most power consuming component in a receiver; as shown in the previews comparison of state-ofthe-art WuRx.

However, this means that this receiver can process amplitude modulated signals only and the architecture calls for a very high selectivity at RF, which will be explained later in detail. This is a result of the behavior of the ED converting all signals directly to baseband without filtering.

The designated transmit distance of our WPAN networks with WuRx is between 5 and $30 \mathrm{~m}$ indoors. In our scenario, the WuRx works at the same frequency as the main transceiver. To reduce hardware complexity, the main transceiver will be used to generate the OOK signal.

As widely known, a higher carrier frequency enables broader bandwidth and therefore a high data rate as well as reduced diffraction loss, smaller antenna size and overall increased level of integration.

However, a higher carrier frequency also increases the path loss and therefore the necessary sensitivity of the receiver. Moreover, additional filtering and amplification at high frequencies is more complex and power hungry. The formula for free space path loss is:

$$
\begin{aligned}
\frac{L_{\mathrm{s}}}{\mathrm{dB}} & =10 \log _{10}\left(\frac{4 \pi d}{\lambda}\right)^{2}=20 \log _{10}\left(\frac{4 \pi f d}{c}\right) \\
& =20 \log _{10}(d)+20 \log _{10}(f)-147.55
\end{aligned}
$$

with the carrier wavelength $\lambda(\mathrm{m})$, the carrier frequency $f(\mathrm{~Hz})$, the speed of light $c\left(\mathrm{~m} \mathrm{~s}^{-1}\right)$ and the link distance $d(\mathrm{~m})$. To calculate the path loss, we consider a direct line of sight and no walls and floors, which is equal to a large room. As shown by (Heereman et al., 2011) the path loss exponent then is smaller than 2 , so for simplicity we can use the simple free space path loss equation formula.

Hence with a carrier frequency of $2.48 \mathrm{GHz}$ and a link distance of $10 \mathrm{~m}$ the path loss would be $70 \mathrm{~dB}$, at $30 \mathrm{~m}$ roughly $80 \mathrm{~dB}$. A better insight of indoor wireless coverage can be found in (Plets et al., 2013). For WLAN at $2.467 \mathrm{GHz}$ and a distance of $1 \mathrm{~m}$ the result is $L_{s}=-40 \mathrm{~dB}$ which is an attenuation of $40 \mathrm{~dB}$.

Inserting the system specific transmit power $P_{\mathrm{TX}}[\mathrm{dB}]$ and the ED sensitivity $S_{\mathrm{ED}}[\mathrm{dB}]$ we can calculate the resulting link margin $L_{\mathrm{M}}[\mathrm{dB}]$. This can be interpreted as the additional gain necessary to be able to detect a signal at the inserted frequency and distance.

$$
\begin{aligned}
\frac{L_{\mathrm{M}}}{\mathrm{dB}}= & P_{\mathrm{TX}}-S_{\mathrm{ED}} \\
& -20 \log _{10}(d)-20 \log _{10}(f)+147.55
\end{aligned}
$$

Setting the link margin to zero and solving the equation for $d$ gives the distance at which the signal can be detected, or at which an interferer can no longer disturb.

$d=10^{\left(P_{\mathrm{TX}}-S_{\mathrm{ED}}-20 \log _{10}(f)+147.55\right) / 20}$

Also, considering the overall gain $G[\mathrm{~dB}]$ and interferer attenuation $A_{\mathrm{I}}[\mathrm{dB}]$ of the system and solving the equation for $A_{\mathrm{I}}[\mathrm{dB}]$ we can calculate the necessary attenuation for an interferer in a dedicated distance.

$$
\begin{aligned}
\frac{A_{\mathrm{I}}}{\mathrm{dB}}= & P_{\mathrm{TX}}-S_{\mathrm{ED}}+G \\
& -20 \log _{10}(d)-20 \log _{10}(f)+147.55
\end{aligned}
$$

Figure 1 shows the ISM and LTE Band allocation for WLAN, Bluetooth, WPAN and LTE Band 7. As shown the ISM Band is used by a lot of participants in different channels. Bluetooth uses $1 \mathrm{MHz}$ channels and frequency hopping so collision with a Bluetooth device should not occur too often and only for short times. WLAN uses 20 or $40 \mathrm{MHz}$ channels, e.g. when a video is streamed the channel is used continuously, this interferer has to be suppressed as much as possible. LTE Band 7 is used for mobile communication. It also uses a high bandwidth and a higher transmit power.

For our test system we choose to use the WPAN channel with fewest interferences which is channel 26 at $2.48 \mathrm{GHz}$. 


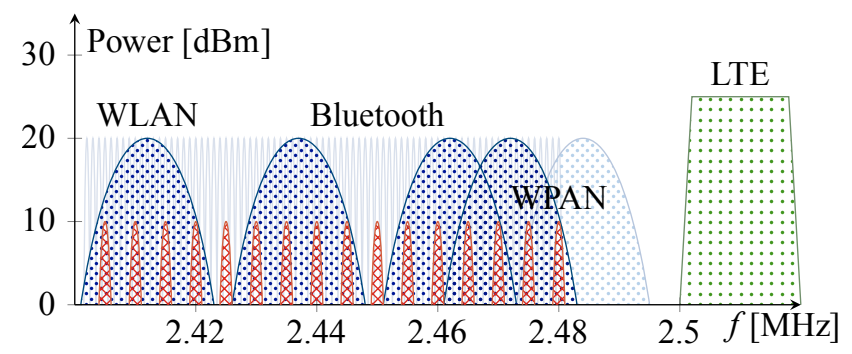

Figure 1. ISM and LTE band 7 allocation.

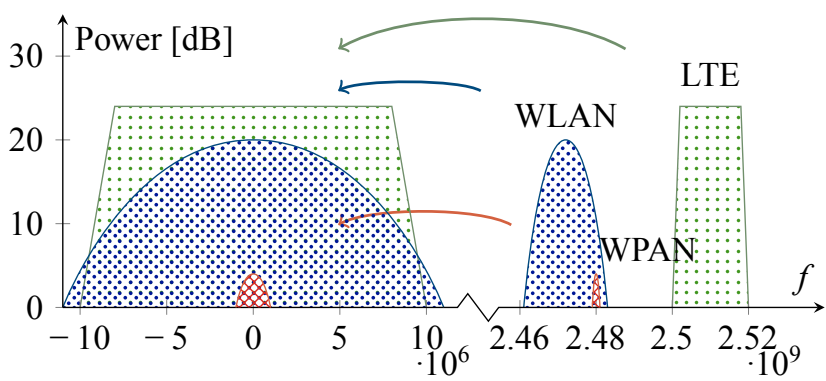

Figure 2. Down conversion of RF signals to baseband by envelop detection with WLAN and LTE communication as interference.

Figure 2 illustrates the down-conversion of the RF band to baseband by an ED containing the wake-up signal on WPAN channel 26 and the two closest interferers, WLAN channel 13 and LTE band 7. As shown in the illustration the interferers potentially have a higher transmit power than our WPAN node, which is specified for the AT68RF233 as $4 \mathrm{dBm}$ without losses due to the balloon, the antenna or mismatch.

As defined in (IEEE 802.11b, 2000), the maximum transmit power of WLAN is $20 \mathrm{dBm}$ and LTE band 7 user equipment and base stations are limited to $24 \mathrm{dBm}$ as defined in (ETSI TS 136 101, 2014) and (ETSI TS 136 104, 2010). Allready mentioned earlier a narrowband filter in the RF band is necessary to suppress interferers and converts down only the band of interest to be able to detect the signal with the ED. Calculating the attenuation needed to suppress WLAN with a transmit power of $20 \mathrm{dBm}$ at a distance of $1 \mathrm{~m}$ for a system using an ED with a sensitivity of $-30 \mathrm{dBm}$ results in approximately $10 \mathrm{~dB}$ and for LTE with $24 \mathrm{dBm}$ transmit power in $13.7 \mathrm{~dB}$, according to Eq. (4).

\section{System design}

\subsection{System setup}

For this work the absolute power consumption of the system is not important since it cannot compete with integrated solutions anyway. The emphasis is on the knowledge gained on how parts influence each other, System feasibility and usability in a real world application.

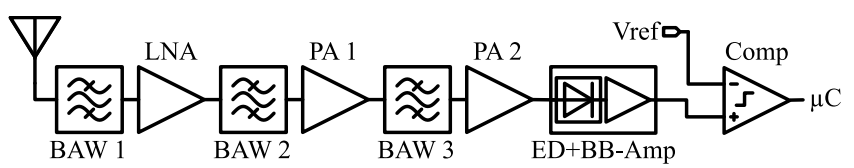

Figure 3. WuRx system concept with overlapping filters to gain an extremely narrowband filtering.

All parts had to be chosen to allow for manual soldering for fast adaption of changes and debugging.

Figure 3 shows the different stages of the WuRx. To achieve a good interferer suppression, we designed the WuRx using multiple bulk acoustic wave (BAW) filters with different pass characteristics, which will be explained later.

The WuRx sensitivity suffers heavily from the additional attenuation of the bandpass filter configuration. Increased gain is the only way to increase the sensitivity, but amplification comes with the cost of power consumption in general.

The LNA SKY67159-396LF from Skyworks has promising features but was not commercially available at the time. Skyworks provided some samples, we decided to not rely on this LNA only and used another amplifier for the 2 and 3 stage. The SKY67159-396LF is a broadband amplifier, that works from $200 \mathrm{MHz}$ to $3800 \mathrm{MHz}$, has a gain of $17 \mathrm{~dB}$ and a very low noise figure of approximately $1 \mathrm{~dB}$.

HMC414, PA1 and PA2, manufactured by Hittite Microwave Products (HMC) designed for frequencies between 2.2 and $2.8 \mathrm{GHz}$ has a gain of up to $20 \mathrm{~dB}$, a noise figure of $6.5 \mathrm{~dB}$ and enable-times in the nanosecond time scale.

Additionally, the output current can be set by dedicated pins and can be reduced as much as possible without influencing the sensitivity.

The ED, which demodulates the wake-up signal by converting the incoming carrier frequency to DC, should be implemented similarly to the way it is done in integrated wakeup receivers. Therefore, the choice is limited to EDs that consist of a diode followed by a RC low pass filter. The chosen detector is the LTC5508 produced by Linear Technology and comes only in combination with a buffer amplifier in one package. It has a frequency operation range of 300 to $7000 \mathrm{MHz}$ and supports data rates up to $2 \mathrm{MHz}$, which is more than sufficient for this work. The buffer amplifier has an output voltage range of around 0.25 up to $1.75 \mathrm{~V}$ and omits the use of additional gain after detection. Unfortunately, the data sheet shows that the output voltage is between 0.15 and $0.4 \mathrm{~V}$ even during the absence of an input signal. For reliable information, the component must be calibrated after it has been implemented in the circuit. The output offset also has influence on the detection threshold for the comparator to work properly. As the data sheet states it has a sensitivity of -32 to $12 \mathrm{dBm}$, but between 2 and $3 \mathrm{GHz}$ the output voltage change at -32 to $-25 \mathrm{dBm}$ input power is very small and measurements have to show how much of that sensitivity range is actually effective. 
Table 1. Data sheet specifications at $25^{\circ} \mathrm{C}, 3.3 \mathrm{~V}, 2.481 \mathrm{GHz}$.

\begin{tabular}{lrr}
\hline & $\begin{array}{r}\text { Current } \\
\mathrm{mA}\end{array}$ & $\begin{array}{r}\text { Gain } \\
\mathrm{dB}\end{array}$ \\
\hline LNA & 45.5 & 17.2 \\
Power Amplifier1 +2 & $494^{*}$ & 34 \\
Envelope Detector & 0.55 & - \\
Comparator & 0.275 & - \\
\hline Total & 540.3 & 51.2 \\
\hline
\end{tabular}

* Absolute power consumption is not focus of this work.

The last active component of the front-end is a comparator which serves as 1-bit ADC. The requirements are very low since the wake-up signal is in baseband at this stage and has a fairly low data rate of $1 \mathrm{kHz}$. It should have a pin to latch the output.

The used comparator is a MAX9141 produced by Maxim Integrated. It is a low power, high speed device and exceeds the needed requirements. The data sheet states a propagation delay of $140 \mathrm{~ns}$, a latch delay of $16 \mathrm{~ns}$ for setup and hold time and a latch propagation delay of $60 \mathrm{~ns}$.

It features an internal hysteresis of about $1.5 \mathrm{mV}$; this ensures clean switching even with slow moving input signals generated by the ED. The threshold at which the comparator switches from 0.3 to $3 \mathrm{~V}$ is adjustable. Its value depends on the dc offset of the ED, the received signal strength od the wake-up signal, the gain of the WuRx and the received signal strength of the interferer.

An extraction of some interesting characteristics of the parts used are listed in Table 1, taken from the data sheets.

\subsection{Narrowband filter}

As mentioned earlier the interferers potentially have a higher power than the wanted signal which leads to the necessity of strong suppression. To achieve the necessary interferer rejection, a combination of multiple BAW filters are used, which have an overlapping frequency range in the designated area. For a cheap WuRx BAW filter will not be feasible, so in the concept of the integrated version the interferer rejection should be addressed by selective amplification and low power filter concepts.

More information about BAW filters can be found in (Mahon and Aigner, 2007). We choose two BAW filters from Triquint, BAW $885033\left(f_{\mathrm{c}}=2.442 \mathrm{MHz}, \mathrm{BAW} 3\right)$ and BAW $885009\left(f_{\mathrm{c}}=2.535 \mathrm{MHz}, \mathrm{BAW} 1 / 2\right)$. The BAW 885033 has a bandwidth of $79 \mathrm{MHz}$ and for the desired frequency of $2.481 \mathrm{GHz}$ it has an insertion loss of 1.7 to $2.2 \mathrm{~dB}$ as shown in Fig. 4. It creates the upper cut-off-frequency for interferers, especially LTE in Germany, starting at $2.5 \mathrm{GHz}$. According to the data sheet the attenuation at $2.5 \mathrm{GHz}$ is around $40 \mathrm{~dB}$ at $25^{\circ} \mathrm{C}$. The BAW 885009 filter used as BAW1/2 shown in Fig. 4 forms the lower cut-off frequency and has a bandwidth

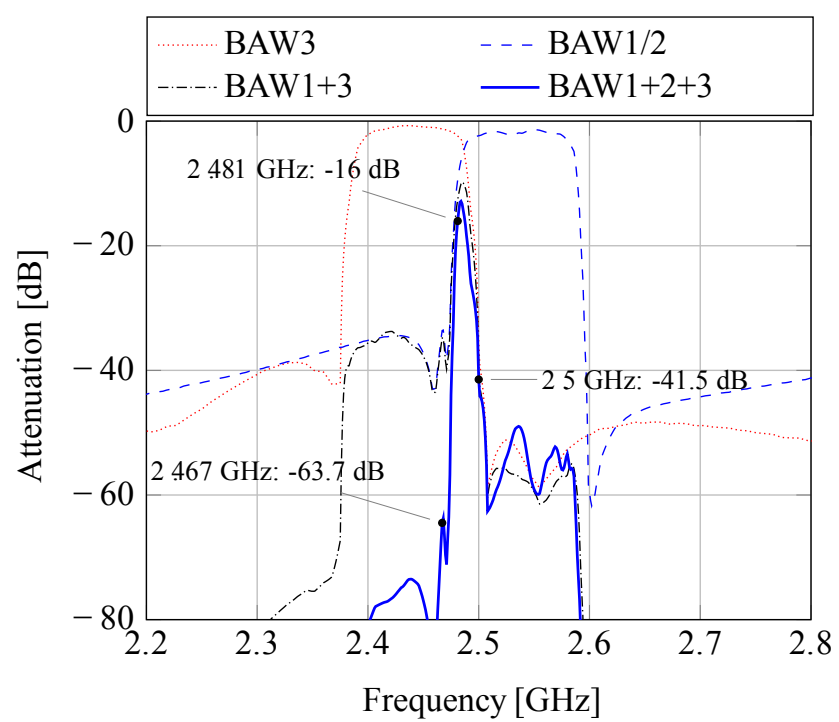

Figure 4. Simulated S21-parameter of the BAW filters and their combinations.

of $70 \mathrm{MHz}$. The desired frequency lies at the lower edge of this filter and is attenuated by about $9.5 \mathrm{~dB}$. Undesired frequencies that go up to $2.473 \mathrm{GHz}$ will be attenuated by at least $33 \mathrm{~dB}$. Unfortunately there were no $S$-parameter files provided for the BAW 885033 so the $S$-parameters of a BAW filter from the same manufacturer, that comes very close to the used one are shown in Fig. 4 for simulation and visualization.

Combining the two filters (BAW1 +3 ) results in the characteristic as shown in Fi. 4. Calculated values result in an attenuation of $12.6 \mathrm{~dB}$ for $2.481 \mathrm{GHz}$, of $34.8 \mathrm{~dB}$ for $2.467 \mathrm{GHz}$ and of $39.1 \mathrm{~dB}$ for $2.5 \mathrm{GHz}$. With a gain of $51.5 \mathrm{~dB}$ this would result in approx. $11 \mathrm{~m}$ distance needed to the next WLAN router and $17 \mathrm{~m}$ to the next LTE device. This is not sufficient for our scenario, as coexistence with at least WLAN should be possible.

Subsequently, an additional filter, BAW2, with a centre frequency at $2.535 \mathrm{GHz}$ was used.

The plots for BAW3, BAW $1 / 2$ and BAW $1+3$ are made using unmatched components to get a general overview of the behavior. In a last step, a matching network was designed to increase performance for the frequency used.

For BAW3 the attenuation at $2.481 \mathrm{GHz}$ is already low and at $2.5 \mathrm{GHz}$ sufficient, so adapting the matching was not necessary. The impedance matching network provided in the data sheet was used, because there were no $S$-parameters provided. For the other filters $S 11$ and $S 22$ were adjusted with available capacitors and inductors for best performance at $2.481 \mathrm{GHz}$. These had to be selected carefully with focus on low resistance to keep losses low and a high series resonance frequency to maintain the desired properties. The result is shown as trace BAW $1+2+3$ in Fig. 4. The attenuation at $2.481 \mathrm{GHz}$ decreased by around 3 to $16 \mathrm{~dB}$, it increased 


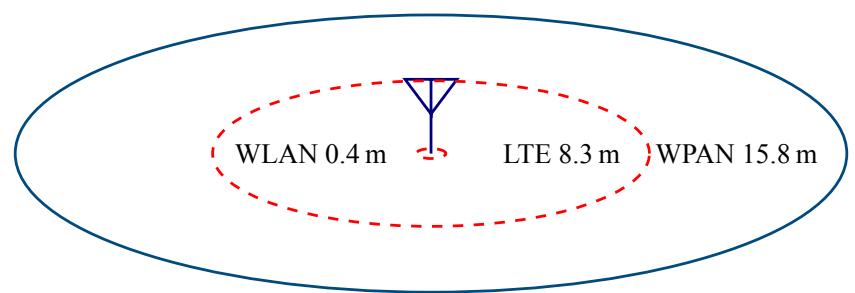

Figure 5. Min. interferer and max. transmitter distance.

to $63.7 \mathrm{~dB}$ at $2.467 \mathrm{GHz}$ and at $2.5 \mathrm{GHz}$ by around 1.5 to $41.5 \mathrm{~dB}$, as marked in Fig. 4. Consequently, the distance to WLAN reduces to $0.7 \mathrm{~m}$ and for LTE to $14.8 \mathrm{~m}$. Adding more attenuation for LTE would have added too much attenuation to the desired signal. With the choosen 3 filter configuration the system will still be error prone for LTE, but sufficiently robust against WLAN.

\subsection{Link margin}

In Fig. 5 the minimal distances for WLAN, LTE and the maximum distance for the transmitter and the WuRX are shown.

Calculating the link margin has been done repeatedly throughout the work to evaluate the results of this approach. The final performance presented here is achieved with simulated data of the architecture and components described before. With a sensitivity of $-25 \mathrm{dBm}$ of the $\mathrm{ED}$, a gain of about $35.2 \mathrm{~dB}$ of the amplifier and filter stage, we get a sensitivity of $-60.2 \mathrm{dBm}$ at $2.481 \mathrm{GHz}$. With an assumed radiated output power of $4 \mathrm{dBm}$ the equation for path loss gives an ideal maximum link distance of around $15.6 \mathrm{~m}$.

This will be reduced by the small output swing of the ED and noise at the input of the ED, which effect the configuration of the threshold voltage of the comparator. Considering the output offset of the ED a reasonable start value for the threshold of the comparator is $280 \mathrm{mV}$ at the output of the $\mathrm{ED}$. The minimum distance is also limited by the ED with an upper limit of $12 \mathrm{dBm}$, that can be detected. This leads to a minimum distance of around $0.2 \mathrm{~m}$.

The input compression of the amplifiers is not be reached at this point with these components. With the maximum radiation power of $20 \mathrm{dBm}$ and an attenuation of $12.5 \mathrm{~dB}$ WLAN signals will not disturb at a distance of around $0.4 \mathrm{~m}$.

Depending on the distance of the base station the radiation power for mobile phones can reach up to $24 \mathrm{dBm}$ as specified in the $3 \mathrm{G}$ standard, to which LTE belongs (3.9G). The uplink frequencies for LTE vary from $2.5 \mathrm{GHz}$ up to $2.57 \mathrm{GHz}$ in Germany. With a total gain of $10 \mathrm{~dB}$ for LTE frequencies, these signals overshadow the desired signal significantly at the same distance to the receiver.

A minimum distance of $8.3 \mathrm{~m}$ to the LTE transmitter is necessary prevent corruption of the wake-up sequence. To coexist closer to LTE, we can adjust the threshold of the com-
Table 2. Measured electric specifications, $3.3 \mathrm{~V}$

\begin{tabular}{lrr}
\hline & $\begin{array}{r}\text { Current } \\
\mathrm{mA}\end{array}$ & $\begin{array}{r}\text { Gain @ 2.481 GHz } \\
\mathrm{dB}\end{array}$ \\
\hline BAW 1+2+3 & - & -16 \\
LNA & 45.5 & 13.35 \\
Power Amplifier 1 & $71.9^{1,2}$ & 12.82 \\
Power Amplifier 2 & $73^{1,2}$ & 12.21 \\
Envelope Detector & 0.55 & - \\
Comparator & 0.165 & - \\
\hline Total & 191 & 22.38 \\
\hline
\end{tabular}

${ }^{1}$ Absolute power consumption is not focus of this work

${ }^{2}$ With optimized output current configuration.

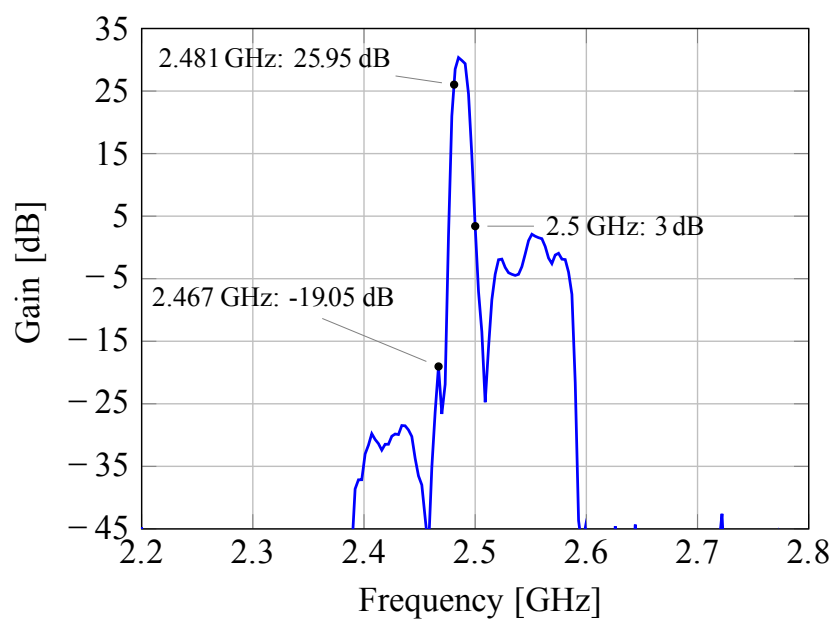

Figure 6. Measured gain of the complete WuRx

parator, which results in a reduced maximum distance of the wake-up receiver to the transmitter.

\section{Implementation and measurements}

To debug the WuRx every stage was soldered and measured successively. As expected, the comparison of simulated and the measured values differ and each stage had to be matched with regard to the measured values again. Some selected measurements are listed in Table 2.

All stages showed lower performance than listed in the data sheet, which is caused by the FR4 substrate of the PCB. The data sheets and application notes are based on Roger substrate and the data sheet values also take board loss deembedding into account. Our measurements include all losses only deembedding the cable losses by calibration.

Getting the HMC414 running was a big challenge because of oscillations which were caused by crosstalk from the output to the input. This could be reduced by inserting a barrier of silver wire between the input and output pin, but still 


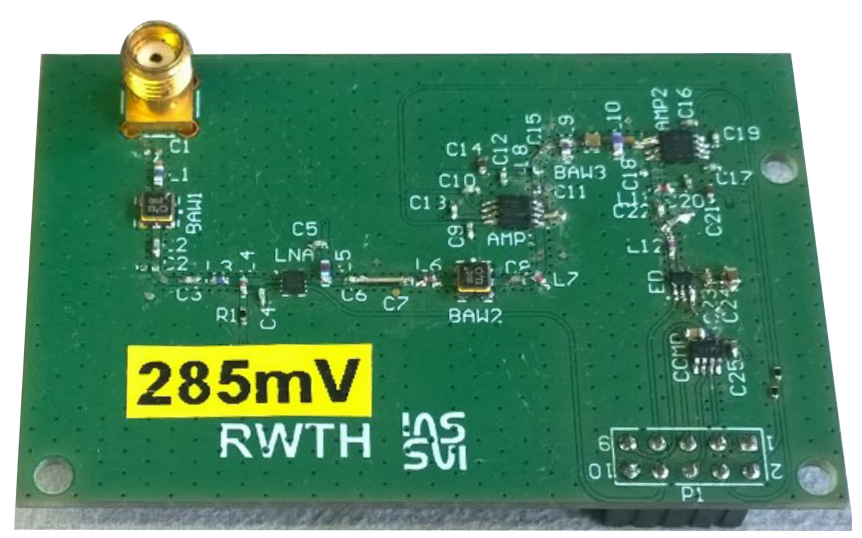

Figure 7. Wake-up receiver PCB.

the maximal gain could not be used. Thus, the gain of the HMC414 is not as high as expected.

The Application note from the (Hittite Microwave Corporation, 2004) addressing this problem helped to solve this issue but was found while debugging. The solutions and improvements shown there could not be implemented on the board to gain the best performance.

Figure 6 shows a measurement of the whole circuit from the SMA connector to the output of the last amplifier. The increase of $3 \mathrm{~dB}$ from single component to whole system measurement can be explained by better matching of the stages to each other than to the measurement equipment.

Considering optimum matching and operating power amplifiers the general functionality of the circuit was tested. For the baseband output voltage of the ED we get a value of about $275 \mathrm{mV}$ with no incoming RF signal. With regard to the input hysteresis and offset of the comparator the voltage is adjusted to make sure it switches only if a signal is present. This threshold is found to be at $284 \mathrm{mV}$. With a signal generator directly connected to the board, the sensitivity at $2.481 \mathrm{GHz}$ is $-20.0 \mathrm{dBm}$. With a gain of $26 \mathrm{~dB}$ this results in an ideal range of about $3 \mathrm{~m}$ with an output power of $4 \mathrm{dBm}$ of the transmitter. For $2.467 \mathrm{GHz}$ we have an attenuation of $19 \mathrm{~dB}$ and for $2.5 \mathrm{GHz}$ a gain of $3 \mathrm{~dB}$.

Table 3 shows the calculated distance using the measured gain and sensitivity values and the measured distance achieved with a signal generator and a transmitter board with an AT86RF233. Interferer rejection was tested by applying the signal from the generator to an antenna.

\section{Conclusions}

In this work, we showed the implementation of a wake-up receiver with commercial components to investigate the needs of such a system. It uses discrete off-the-shelf components and was built on a FR4 PCB to have the possibility to measure and analyze the state of the signal after every step. It
Table 3. Calculated and measured range and interferer rejection.

\begin{tabular}{lrrrr}
\hline & $\begin{array}{r}P_{\text {out }} \\
(\mathrm{dBm})\end{array}$ & & $\begin{array}{r}\text { calc. } \\
(\mathrm{m})\end{array}$ & $\begin{array}{r}\text { meas. } \\
(\mathrm{m})\end{array}$ \\
\hline AT68RF233 & 2.7 & $\max$ & 2.6 & 2 \\
Signal generator & 4 & $\max$ & 3 & 3 \\
WLAN & 20 & $\min$ & 0.1 & 0.3 \\
LTE & 20 & $\min$ & 1.35 & 1.6 \\
\hline
\end{tabular}

will be mounted on a board with a RF transceiver and a microcontroller.

Due to the lack of selectivity of the components used in the circuit, high-Q bandpass filtering had to be implemented to make sure that the receiver works reliably in the $2.4 \mathrm{GHz}$ band. These three successive BAW filters were used to form a narrow bandpass filter and reject even strong out-of-band interferers. The increased losses had to be compensated with additional gain stages. We designed the circuit with a sensitivity of $-60 \mathrm{dBm}$, while still being able to detect a signal in close approximation to interferers sources.

Due to mismatch and oscillation problems, measurements showed a reduced sensitivity of only $-47 \mathrm{dBm}$. However, the attenuation of out-of-band interferers stayed in the same relation to the gain for the desired signal.

This work shows an implementation of an envelopedetection-based WuRx with off-the-shelf components which fulfills the requirements of a real-world application. The further development will be the implementation of a network with this WuRx and investigate how the AT86RF233 can be used to generate the wake-up signal, to investigate the system network setup. The goal of this work was to get better insight in the overall system and the obstacles, gained knowledge by the development is included to the integrated designs.

Data availability. The gain simulation and measurement results are available at Zenodo (Arndt, 2017).

Competing interests. The authors declare that they have no conflict of interest.

Acknowledgements. The authors acknowledge the support of the German Federal Ministry of Education and Research (BMBF) through the "TreuFunk" project (FKZ: 16KIS0234).

Edited by: J. Anders

Reviewed by: two anonymous referees 


\section{References}

Arndt, J.: Implementation of envelope detection based Wake-Up Receiver for IEEE 802.15.4 WPAN from off-the-shelf components,https://doi.org/10.5281/zenodo.582796, 2017.

Atmel: AT86RF233 Low Power, 2.4 GHz Transceiver for ZigBee, RF4CE, IEEE 802.15.4, 6LoWPAN, and ISM Applications, http://ww1.microchip.com/downloads/en/DeviceDoc/ Atmel-8351-MCU_Wireless-AT86RF233_Datasheet.pdf, 2014.

ETSI TS 136 101, T. S.: ETSI TS 136101 V12.5.0 (2014-11), LTE;Evolved Universal Terrestrial Radio Access (E-UTRA); User Equipment (UE) radio transmission and reception (3GPP TS 36.101 version 12.5.0 Release 12), 2014.

ETSI TS 136 104, T. S.: ETSI TS 136104 V9.4.0 (2010-07), LTE; Evolved Universal Terrestrial Radio Access (E-UTRA); Base Station (BS) radio transmission and reception (3GPP TS 36.104 version 9.4.0 Release 9), 2010.

Griggs, J. D.: Ultra-Low Power Wake up Receiver for Medical Implant Communications Service Transceiver, Ph.D. thesis, North Carolina A\&T State University, 2012.

Gutierrez, J. A., Callaway, E. H., and Barrett, R.: IEEE 802.15.4 Low-Rate Wireless Personal Area Networks: Enabling Wireless Sensor Networks, IEEE Standards Office, New York, NY, USA, 2003

Hambeck, C., Mahlknecht, S., and Herndl, T.: A $2.4 \mu \mathrm{W}$ Wake-up Receiver for wireless sensor nodes with $-71 \mathrm{dBm}$ sensitivity, in: 2011 IEEE International Symposium of Circuits and Systems (ISCAS), 534-537, https://doi.org/10.1109/ISCAS.2011.5937620, 2011.

Heereman, F., Joseph, W., Tanghe, E., Plets, D., and Martens, L.: Prediction of range, power consumption and throughput for IEEE $802.11 \mathrm{n}$ in large conference rooms, in: Proceedings of the 5th European Conference on Antennas and Propagation (EUCAP), 692-696, 2011
Hittite Microwave Corporation, A. D.: Designing w/The HMC414MS8G PA Utilizing a Low Cost Laminated Printed Circuit Board, 2004.

Huang, X., Rampu, S., Wang, X., Dolmans, G., and de Groot, H.: A $2.4 \mathrm{GHz} / 915 \mathrm{MHz} 51 \mu \mathrm{W}$ wake-up receiver with offset and noise suppression, in: 2010 IEEE International Solid-State Circuits Conference - (ISSCC), 222-223, https://doi.org/10.1109/ISSCC.2010.5433958, 2010.

IEEE 802.11b: IEEE Standard for Information Technology Telecommunications and information exchange between systems - Local and Metropolitan networks - Specific requirements - Part 11: Wireless LAN Medium Access Control (MAC) and Physical Layer (PHY) specifications: Higher Speed Physical Layer (PHY) Extension in the $2.4 \mathrm{GHz}$ band, IEEE Std 802.11b1999, 1-96, https://doi.org/10.1109/IEEESTD.2000.90914, 2000.

Mahon, S. and Aigner, R.: Bulk Acoustic Wave Devices - Why, How, and Where They are Going, in: CS MANTECH Conference, 2007.

Oetting, J.: A Comparison of Modulation Techniques for Digital Radio, IEEE T. Commun., 27, 1752-1762, https://doi.org/10.1109/TCOM.1979.1094370, 1979.

Pletcher, N. M., Gambini, S., and Rabaey, J. M.: A $2 \mathrm{GHz}$ $52 \mu \mathrm{W}$ Wake-Up Receiver with $-72 \mathrm{dBm}$ Sensitivity Using Uncertain-IF Architecture, in: 2008 IEEE International SolidState Circuits Conference - Digest of Technical Papers, 524633, https://doi.org/10.1109/ISSCC.2008.4523288, 2008.

Plets, D., Joseph, W., Vanhecke, K., Tanghe, E., and Martens, L.: Simple Indoor Path Loss Prediction Algorithm and Validation in Living Lab Setting, Kluw. Commun., 68, 535-552, https://doi.org/10.1007/s11277-011-0467-4, 2013. 